\section{THE IMPACT OF COLLABORATION BETWEEN PHYSICIANS, NURSES, AND CLINICAL PHARMACISTS IN REDUCING MEDICATION PRESCRIBING ERRORS IN KING ABDULLAH SPECIALIZED CHILDREN'S HOSPITAL ER}

Milfi Al-Onazi, Rahayu Rasheed, Faith Vabaza, Ahmed Othman, Leizl Villanueva, Ghada Mardawi. Emergency Department, King Abdullah Specialist Children Hospital

\subsection{6/bmjoq-2019-PSF.64}

Background Medication errors remain the leading cause of mortality and morbidity in the pediatric emergency room (ER) across the world. The Institute of Medicine (IOM) has noted that this is due to the unique needs of the pediatric population compared with adults. With the introduction of the Computerized Physician Order Entry (CPOE) system in the National Guard Health Affairs (NGHA), there was a decrease in medication errors resulting from prescription. Inevitably, the CPOE did not eliminate all prescription errors. The King Abdullah Specialist Children Hospital Emergency Department reviews all safety reports (SRS) as part of the daily key performance indicators meeting with the ER Chairman, nurse managers, and charge nurses. The SRS report from 2016 showed a total of 102 errors. Medication administration is a process that commences with prescription by physicians, then dispensing by the clinical pharmacists, and finally administration by nurses. The NGHA adheres to the 'swiss cheese model' recommended by its Safety Medication Program; therefore, the majority of these errors were 'near miss', meaning they were caught in the process before reaching the patient. The aim of this project was, therefore, to reduce the rate of prescription errors by $60 \%$ by the end of 2018 .

Methods This project was done in the KASCH Emergency Department with a total of 65 beds. Total visits can be up to 500 patients in 24 hours, especially during the winter season. PDSA (plan-do-study-act) methodology was applied in this quality improvement project. A collaborative team was formed led by the ER Chairman. A series of meetings were held. Cycle 1: team formation, brainstorming, and data validation and analysis. The following themes were identified: dose incorrect, frequency incorrect, and allergy override. The following medications were also identified with frequent errors: paracetamol, dexamethasone, and antibiotics. Cycle 2 focused on establishing order sets, pre-calculated prescription doses based on weight on BestCare. Cycle 3: allergy awareness campaign to all clinicians. PDSA 4 focused on the accuracy of reporting errors follow-up and action plans to be implemented and documented.

Results The overall results showed that there were 28 ER prescription errors in the year 2018 compared with 102 in 2016, reflecting a reduction of $73 \%$.

Conclusion The success of this project was evidence that collaboration among all clinicians involved in medication administration can greatly decrease medication errors. This project aims to spread to all areas of $\mathrm{KASCH}$ to address the challenge of medication errors.

\section{OUTBREAK OF MEASLES AND MUMPS IN THE} NATIONAL GUARD POPULATION IN RIYADH, 2018

Rawabi Al Mulhem, Almunif Sara, El-Saed Aiman, Alshamrani Majid, Balkhy Hanan. Infection Control Department National Guard Health Affairs

10.1136/bmjoq-2019-PSF.65
Background Measles, mumps, and rubella (MMR) vaccine is a safe combined vaccine that is considered one of the most effective protective measures against three separate diseases: measles, mumps, and rubella (German measles). During 2018, there were reports of an increased number of measles cases in Saudi Arabia and globally. The objective of the current study was to confirm the presence of such an increase among the National Guard population and to assess if mumps and rubella were also affected.

Methods Communicable diseases are regularly reported from the National Guard population in all regions to the public health section of the Infection and Prevention Control (IPC) department at Riyadh. Retrospective review of prospectively collected information about the three diseases prevented by MMR was conducted in 2019. This covered the period between 2008 and 2018 .

Results During the study period, a total of 48 patients with measles, 84 with mumps, and 20 with rubella had been reported to the IPC department. Compared with the previous 10 years, the number of patients with measles increased from an average of 3.7 to 11 (197\% increase). Compared with the previous 10 years, the number of patients with mumps increased from an average of 5.6 to 28 (400\% increase). Compared with the previous 10 years, the number of patients with rubella increased from an average of 1.8 to $2(11 \%$ increase).

Conclusion We are confirming an outbreak of both mumps and measles. This may indicate that the increase is related to a problem with MMR coverage or effectiveness. Further research is required to confirm the possible causes of this outbreak: missing immunization, shortness of vaccine, misconception about the link between the MMR vaccine and autism, vaccine-induced disease, and pockets of unvaccinated immigrants. Additionally, there is an urgent need to increase public awareness of the MMR vaccine.

\section{IMPROVEMENT IN THE OUTCOME OF THE VIRAL HEPATITIS PREVENTION PROGRAM AT NATIONAL GUARD HOSPITALS (RIYADH) IN 2018}

Al Eniz Nouf, Abrar Turkstani, Kholoud Al Ameer, Sara Almunif, Majed Al Shamrani, Hanan Balkhy, Aiman Ramadan. Infection Prevention and Control King Abdulaziz Medical City

\subsection{6/bmjoq-2019-PSF.66}

Background The public health team of the Infection Prevention and Control (IPC) department conducted a systemic evaluation in 2017 for patients diagnosed with hepatitis B or C between 2013 and 2016. The findings showed high rates of loss to follow-up and unreachable viral hepatitis $\mathrm{B}$ and $\mathrm{C}$ infected cases. Loss to follow-up has been shown to be a major obstacle for management of patients with hepatitis. The objective of the current study was to improve the rate of case identification and follow-up management.

Methods To improve the rate of case identification and follow-up management, the guidelines for viral hepatitis were upgraded in 2018 by IPC staff after active engagement of head and treating physicians of relevant departments and hospital executives. Additionally, key performance improvement (KPI) metrics were established: loss to follow-up (unable to reach patient) and increase in management initiation (able to reach and counsel patient). Data for 